\title{
Heterolytic Scission of Hydrogen Within a Crystalline Frustrated Lewis Pair
}

\section{Bowden, Mark E.}

2020-10-19

Bowden , M E , Ginovska , B , Jones , M O , Karkamkar , A J , Ramirez-Cuesta , A J , Daemen , L L , Schenter , G K , Miller , S A , Repo , T , Chernichenko , K, Leick, N , Martinez , M B \& Autrey , T 2020 , ' Heterolytic Scission of Hydrogen Within a Crystalline

Frustrated Lewis Pair ' , Inorganic Chemistry , vol. 59 , no. 20 , pp. 15295-15301 . https://doi.org/10.1021/acs.inorgcl

http://hdl.handle.net/10138/340998

https://doi.org/10.1021/acs.inorgchem.0c02290

unspecified

acceptedVersion

Downloaded from Helda, University of Helsinki institutional repository.

This is an electronic reprint of the original article.

This reprint may differ from the original in pagination and typographic detail.

Please cite the original version. 


\section{Heterolytic Scission of Hydrogen Within a Crystalline Frustrated Lewis Pair}

Mark E. Bowden a, ${ }^{a}$, Bojana Ginovskab, ${ }^{\text {, }}$, Martin Owen Jones, d, Abhijeet J. Karkamkarb, Anibal J. Ramirez-Cuesta ${ }^{e}$, Luke L. Daemene, Gregory K. Schenter ${ }^{b}$, Seth A. Millerb, Timo Repof, Konstantin Chernichenkof, ${ }^{\text {1 Noemi Leick }}{ }^{\mathrm{g}}$, Madison B. Martinez ${ }^{\mathrm{g}}$, and Tom Autrey ${ }^{\mathrm{b}}$

a. Environmental Molecular Sciences Laboratory, Pacific Northwest National Laboratory, PO Box 999 Richland, WA 99352, USA

b. Physical and Computational Sciences Directorate, Pacific Northwest National Laboratory, PO Box 999 Richland, WA 99352, USA

c. ISIS Neutron and Muon Spallation Facility, STFC, RAL, Didcot OX11 OQX, UK

d. St Andrews University, St Andrews, Fife KY16 9AJ, Scotland UK

e. $\quad$ Spallation Neutron Source, Oak Ridge National Laboratory, TN 37830, USA

f. Department of Chemistry, University of Helsinki, P.O. Box 55, 00014 Helsinki, Finland

g. National Renewable Energy Laboratory, 15013 Denver West Parkway, Golden, CO 80403, USA

*mark.bowden@pnnl.gov, bojana.ginovska@pnnl.gov

${ }^{1}$ Present address: Janssen Pharmaceutica N.V., API Small Molecule Development, Turnhoutseweg 30, 2340 Beerse, Belgium 


\begin{abstract}
We report the heterolysis of molecular hydrogen under ambient conditions by the crystalline frustrated Lewis pair (FLP) 1-\{2-[bis(pentafluorophenyl)boryl]phenyl\}-2,2,6,6tetramethylpiperidine (KCAT). The gas-solid reaction provides an approach to prepare the solvent-free, polycrystalline ion pair KCATH2 through a single crystal to single crystal transformation. The crystal lattice of KCATH2 increases in size relative to the parent KCAT by approximately 2\%. Microscopy was used to follow the transformation of the highly colored red/orange KCAT to the colorless KCATH 2 over a period of 2 hours at $300 \mathrm{~K}$ under a flow of $\mathrm{H}_{2}$ gas. There is no evidence of crystal decrepitation during hydrogen uptake. Inelastic neutron scattering employed over a temperature range from $4-200 \mathrm{~K}$ did not provide evidence for the formation of polarized $\mathrm{H}_{2}$ in a pre-cursor complex within the crystal at low temperatures and high pressures. However, at $300 \mathrm{~K}$ the INS spectrum of KCAT transformed to the INS spectrum of KCATH2. Computational methods suggest that the driving force is more favorable in the solid state compared to the solution or gas phase, but the addition of $\mathrm{H}_{2}$ into the KCAT crystal is unfavorable. Ab Initio methods were used to calculate the INS spectra of KCAT, KCATH2 and a possible precursor complex of $\mathrm{H}_{2}$ in the pocket between the $\mathrm{B}$ and $\mathrm{N}$ of crystalline KCAT. Ex-situ NMR showed that the transformation from KCAT to KCATH2 is quantitative and our results suggest that the hydrogen heterolysis process occurs via $\mathrm{H}_{2}$ diffusion into the FLP crystal with a rate-limiting movement of $\mathrm{H}_{2}$ from inactive positions to reactive sites.
\end{abstract}

\title{
Introduction
}

Catalytic activation of molecular hydrogen is critical for the efficient upgrading of energy carriers ranging from conventional hydrocarbons to renewables such as biomass. While platinum group metals ${ }^{1}$ are recognized as efficient catalysts for $\mathrm{H}_{2}$ activation, there has been significant progress in the development of non-precious metal catalysts for hydrogen activation..$^{2-5}$ In parallel there has been an interest in the ability of non-metal catalysts to activate $\mathrm{H}_{2}$ in catalytic reactions. Frustrated Lewis pairs (FLPs), ${ }^{6}$ comprised of Lewis acidic borane and Lewis basic amine or phosphine, activate hydrogen efficiently and have been demonstrated to reduce a wide range of substrates in the solution phase. FLPs are of interest as they provide an approach to heterolytically activate $\mathrm{H}_{2}$ catalytically at moderate and often ambient temperatures and pressures, without recourse to expensive transition metals and complex nanoscale composites.

FLP catalysts, almost exclusively, activate $\mathrm{H}_{2}$ as solvated molecular systems and thus have been investigated predominately in homogeneous catalytic processes. The work of O'Hare et al. ${ }^{7}$ in 2016, demonstrating $\mathrm{H}_{2}$ activation by silica supported BCF as a Lewis base, initiated the activity in $\mathrm{H}_{2}$ activation by solid state FLPs that has followed since. Qu et al. ${ }^{8}$ reviewed 
FLP systems which have separate acidic and basic molecules with at least one of these in the solid state during activation. Computational studies have suggested solid state $\mathrm{H}_{2}$ activation by Lewis acid-base pairs incorporated into MOF structures, ${ }^{9-11}$ however, experimental observations have been limited. In one recent example, the use of solid physical mixtures of FLPs has been shown to be an effective strategy for achieving frustration for molecular pairs that have insufficient steric hindrance to prevent adduct formation in solution. ${ }^{12}$ These solid mixtures have been reported to react with hydrogen to form the corresponding ion pairs, in what was considered to be a partially molten environment at the interface with sufficient molecular flexibility to activate $\mathrm{H}_{2}$ that had diffused there. Ozin et al. have demonstrated hydrogen activation at the surface of rhombohedral $\operatorname{In}_{2} \mathrm{O}_{3-\mathrm{x}}(\mathrm{OH})_{\mathrm{y}}$, and used this to catalyze various hydrogenation reactions. ${ }^{13,14}$ Their work showed how surface analogs of FLPs are generated where terminally bonded surface hydroxide groups act as Lewis bases and the coordinately unsaturated surface indium site acts as a Lewis acid. A research interest of ours is to understand how $\mathrm{H}_{2}$ is activated in the 'pocket' of an FLP. We have measured the activation barrier in the solution phase and found the enthalpic barrier to be quite low and apparently controlled by diffusion of $\mathrm{H}_{2}$ through solution into the activated pocket. ${ }^{15}, 16$ Our hypothesis was that in the solid phase the heterolysis of $\mathrm{H}_{2}$ would be slower assuming a higher barrier for rearrangement of bonding in the solid state to optimize the transition state structure for heterolysis of $\mathrm{H}_{2}$.

The goal of the work was to observe polarization of the $\mathrm{H}_{2}$ molecule in a precursor complex, the "pocket" of an FLP, at low temperature by inelastic neutron scattering (INS) spectroscopy, then warm the crystalline material to room temperature and follow the heterolysis reaction. The heterolysis of $\mathrm{H}_{2}$ by the acid-base pair is subtly different than the ligand exchange reactions on organometallic compounds reported by Brookhart and Weller. Brookhart and co-authors ${ }^{17}$ demonstrated that the non-porous iridium molecular complex, $\left\{\mathrm{C}_{6} \mathrm{H}_{3}-2,6-\left[\mathrm{OP}-\left(\mathrm{C}_{6} \mathrm{H}_{2}\left(\mathrm{CF}_{3}\right)_{3}-2,4,6\right)_{2}\right]_{2}\right\} \mathrm{Ir}-\left(\mathrm{N}_{2}\right)$, underwent a single crystal to single crystal (SCSC) transformation to form a dihydrogen complex on exposure to $\mathrm{H}_{2}$, and provided a scaffold for the catalytic transformation of $\mathrm{CH}_{2}=\mathrm{CH}_{2}$ to ethane. This followed work by Bianchini $e t$ al. ${ }^{18}$ who used an Ir tripodal polyphosphine complex in the solid state to catalytically hydrogenate ethene. Similarly, Weller et al. ${ }^{19}$ showed that solid rhodium bis-phosphine complexes would undergo solid-gas ligand exchange reactions to become active catalysts. These workers showed how SC-SC chemistry can be used to synthesize $\sigma$-alkane complexes ${ }^{20}$, which are typically difficult to prepare otherwise because the weak $\mathrm{M} \cdots \mathrm{H}-\mathrm{C}$ interactions are easily replaced by solvent coordination. They were also able to demonstrate how $\mathrm{C}-\mathrm{H}$ bonds are activated according to their metal interactions, leading to selective deuteration ${ }^{21}$ of exo- hydrogens in coordinated norbornane by exposing crystals to $\mathrm{D}_{2}$ gas at room temperature. 
Here we report heterolytic hydrogen scission by crystalline 1-\{2[bis(pentafluorophenyl)boryl]phenyl\}-2,2,6,6-tetramethylpiperidine (KCAT) at ambient temperatures and pressures to quantitatively yield the corresponding zwitterionic pair. The single crystal to single crystal (SC-SC) transformation provides the first demonstration of a non-metal example of $\mathrm{H}_{2}$ heterolysis by a unimolecular FLP in the solid state. Single crystal and powder X-ray diffraction were used to determine the structure and lattice expansion on hydrogen uptake to form the ion pair (KCATH2). We use a combination of temperature programmed desorption (TPD) and inelastic neutron scattering (INS) to demonstrate that there is little molecular hydrogen solubility within the solid FLP prior to heterolytic dissociation and no evidence for a precursor, polarized- $\mathrm{H}_{2}$, intermediate phase. Optical and NMR measurements were used to confirm the quantitative conversion of KCAT to KCATH2 at ambient temperatures. First principle calculations were used to determine the free energy changes for incorporation of $\mathrm{H}_{2}$ into the crystal lattice, for the reaction to the zwitterion, and to model INS spectra for comparison with experiment.

\section{Experimental and Computational Methods}

We employed an extensive range of spectroscopic and diffraction experiments described in the introduction, as well as DFT and molecular dynamics calculations. These followed common laboratory practice, and details of the methods used are given in the supporting information. All sample manipulations were carried out using glove boxes or other protective atmosphere techniques to avoid reaction with water or other atmospheric gases.

\section{Results}

\section{NMR Spectroscopy}

The quantitative transformation of the solid-state KCAT to KCATH2 was undertaken by filling the headspace above $c a .20 \mathrm{mg}$ of KCAT in an NMR tube with $\mathrm{H}_{2}$ gas at ambient pressure. The KCAT sample was a coarse crystalline powder comprising particles approximately $50-200 \mu \mathrm{m}$ in size. Over the course of 2 hours, the orange/red crystals of KCAT in the NMR tube were observed to lose their color, indicating a transformation to the colorless compound KCATH2 (Fig. 1a). The reaction was confirmed by ${ }^{11} \mathrm{~B}$ NMR spectroscopy after dissolving the product in bromobenzene (Fig. 1b) which shows the ${ }^{1} \mathrm{H}$ coupled doublet resonance at -22.5 ppm characteristic of the ion pair. The broad resonance at +62 ppm displayed by KCAT (published in earlier work ${ }^{22}$, ESI) was no longer detected. 
a)
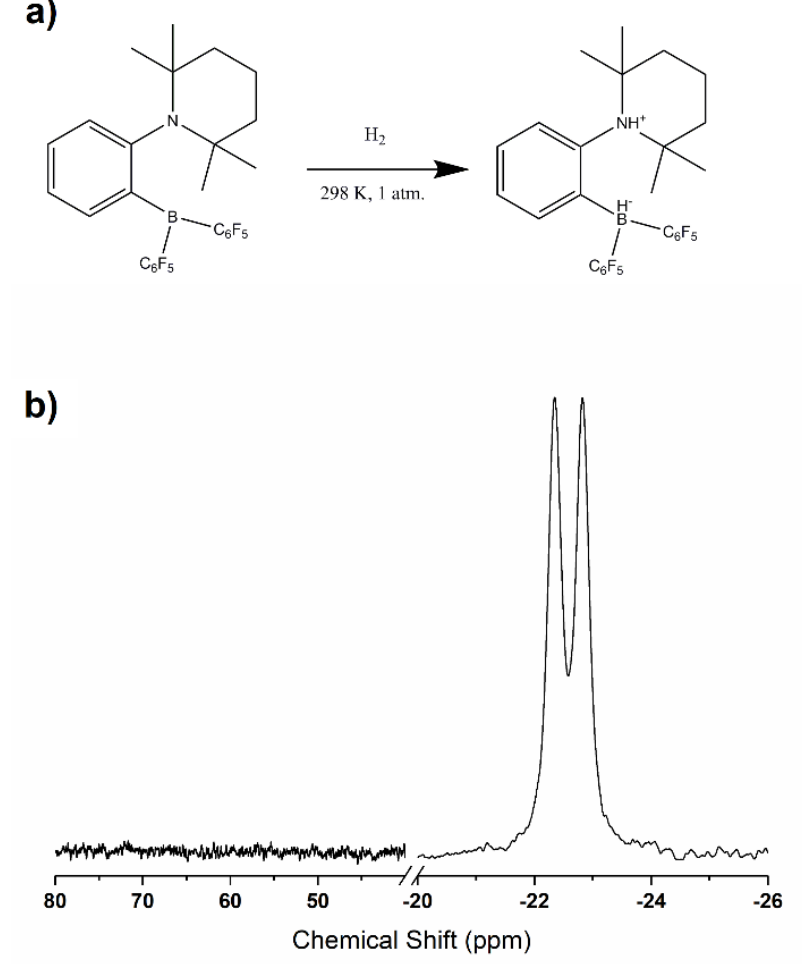

Figure 1. a) KCAT and KCATH2 structures; b) ${ }^{11} \mathrm{~B}$ NMR (in bromobenzene) of KCAT after reaction with $\mathrm{H}_{2}$ gas.

Single crystal and powder X-ray diffraction

Powder X-ray diffraction (Fig. S1) of KCAT and KCATH2 indicated that both the reactant and product were crystalline. The reactant pattern matched that calculated from the crystal structure of KCAT, ${ }^{22}$ but the previously published structure for KCATH2 has entrained solvent and is therefore not suitable for comparison. However, our solid-gas reaction resulted in crystals of KCATH2 free of solvent which were suitable for structure solution by single crystal diffraction. This suggests the reaction is a single crystal - single crystal transformation. The structure we obtained (Fig. 2) has a molecular geometry similar to the solvent-containing crystal. Selected bond distances and angles are tabulated (Table S2) to show how the environments around $\mathrm{N}$ and $\mathrm{B}$ change upon reaction. There is a small (ca. 3.5 $\%$ ) increase in $\mathrm{N}-\mathrm{C}$ bond distances, but the angles around $\mathrm{N}$ do not change significantly because of the more rigid piperidine residue and the presence of the lone pair in KCAT. The increase in B - C bond distances upon heterolysis were slightly larger (ca. $4-6 \%)$ and the geometry around B changed from approximately planar triangular to tetrahedral as expected. This changes the orientations of the pentafluorophenyl rings and consequently some of the intermolecular packing interactions. It is remarkable that the compound remains as a single crystal during this transformation. Further discussion of the structural differences 
between KCATH2 with and without solvent is given in the SI.

The lattice parameters show that KCATH2 has a unit cell volume $2.1 \%$ larger than KCAT. Single crystal measurements of KCAT were also undertaken at temperatures between 100 and $300 \mathrm{~K}$ to detect any phase transition. The structures obtained were the same as the published structure 22 without any phase transition or unusual thermal expansion (Fig. S3).

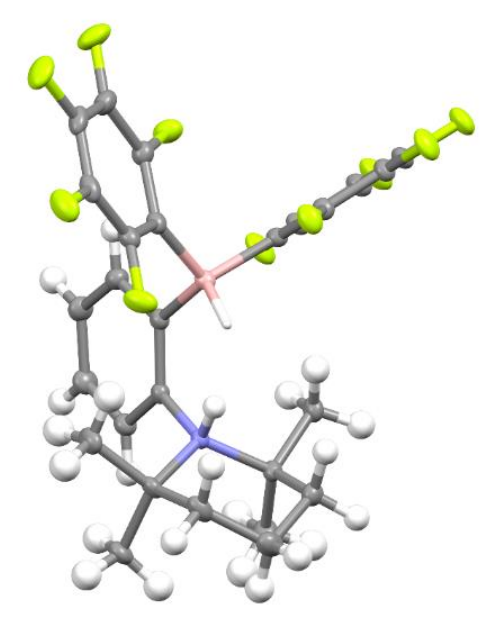

Figure 2. Structure of solvent-free KCATH2 obtained from single crystal XRD. C atoms are grey, $\mathrm{F}$ green, $\mathrm{N}$ blue, $\mathrm{B}$ pink, and $\mathrm{H}$ white.

\section{Optical Microscopy}

Optical microscopy was used to dynamically observe the hydrogen uptake in KCAT. Large crystals of KCAT were grown by slow evaporation from heptane and placed under $\mathrm{N}_{2}$ in a custom gas-tight cell with a microscope slide affixed to one face. After locating a suitable mass of crystals, $\mathrm{H}_{2}$ gas was flowed through the cell at 1 bar pressure and images taken every 30 s, with typical images presented in Fig. 3. These images demonstrated that the external features of the crystal are unaffected by the transformation to the ion pair, with no evidence for decrepitation of hydrogenated material from the host crystal. The most significant change observed was the color change, which occurred slowly throughout most of the crystal. Although the color change was not perfectly homogeneous, we did not observe a sharp reaction front progressing from the outside surface as seen by Weller. ${ }^{19}$ Some portions of the crystal reacted faster than others, but the boundaries between these regions were diffuse and therefore there did not appear to be a clear preference for surface or interior sites. 

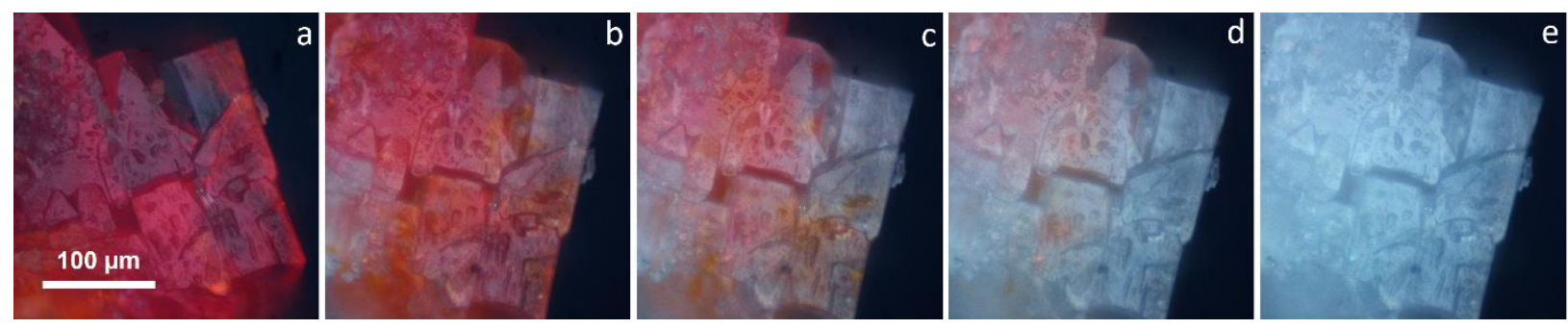

Figure 3. Optical micrographs of KCAT crystals exposed to $\mathrm{H}_{2}$ gas for a) 0 min, b) $55 \mathrm{~min}, \mathrm{c}$ ) 85 min, d) 112 min, e) 150 min.

The volume expansion predicted by the crystal structures was accommodated without any obvious changes to the size and shape of crystals. The crystal under observation moved once between successive frames (between Figs $3 a$ and $3 b$ ) and the field of view was adjusted. However, the retention of the overall integrity of the crystal is surprising given the structural changes which result from the formation of the ion pair.

\section{Inelastic Neutron Scattering}

Investigation of the reaction was undertaken using INS to provide insight into the chemical environment of $\mathrm{H}_{2}$ prior to formation of the ion pair. We were especially interested to see whether any weakly bound $\mathrm{H}_{2}$ between the boron and nitrogen centers could be observed, as suggested by theoretical studies of related systems. ${ }^{23-25}$ Although multiple INS experiments were performed at a variety of temperatures and pressures (see Supporting Information) no unambiguous evidence for dihydrogen, polarized or otherwise, was obtained. For example, the INS spectra measured at $10 \mathrm{~K}$ for KCAT before and after exposure to ca. 1 equivalent $\mathrm{H}_{2}$ at up to $200 \mathrm{~K}$ were indistinguishable (Fig. 4). However, exposure at $300 \mathrm{~K}$ resulted in transformation to the ion pair and a concomitant change in the INS spectrum.

\section{Computational Modelling}

DFT calculations were done for the periodic system, with a cell containing 4 FLP molecules, replicated in XYZ directions for a bulk model. The models were built from the experimentally determined crystal structures reported here. All calculations were done with the CP2K program, ${ }^{26}$ using PBE functional ${ }^{27}$ with Grimme's D2 dispersion correction. ${ }^{28,}{ }^{29}$ DZVP basis set and Goedeker-Hutter pseudopotentials ${ }^{30}$ consistent with the PBE functional were employed. Details of the methods and the approach are provided in the SI (page 9). The energy difference between the FLP and ion pair revealed a strong driving force for the heterolysis with $\Delta E=-135 \mathrm{~kJ} \mathrm{~mol}^{-1} \mathrm{H}_{2}$ and a free energy change at $300 \mathrm{~K}$ of $\Delta A=-125 \mathrm{~kJ} \mathrm{~mol}^{-}$ ${ }^{1} \mathrm{H}_{2}$. However, the presence of molecular $\mathrm{H}_{2}$ within the KCAT lattice was energetically disfavored. Calculations after addition of four different $\mathrm{H}_{2}$ molecules near each of the four 
$\mathrm{B} \cdots \mathrm{N}$ sites in a symmetry-free KCAT unit gave a free energy change after relaxation of $\Delta A=$ $50 \mathrm{~kJ} \mathrm{~mol}^{-1} \mathrm{H}_{2}$, and an electronic energy change of $\Delta E=33 \mathrm{~kJ} \mathrm{~mol}^{-1} \mathrm{H}_{2}$. The electronic energy for individual $\mathrm{H}_{2}$ molecules (Table 1) spanned a range of $\Delta E=+26$ to $+39 \mathrm{~kJ} \mathrm{~mol}^{-1}$. The most stable structure was found to have $\mathrm{H}_{2}$ in a position far from the active $\mathrm{B} \cdots \mathrm{N}$ site and interacting with the $\pi$ system of the pentafluorophenyl rings (Fig. S9, panel 1). A second $\mathrm{H}_{2}$ molecule (site 2, Table 1), although $6 \mathrm{~kJ} \mathrm{~mol}^{-1}$ higher in energy, showed evidence of interaction with nearby $\mathrm{B}$ and $\mathrm{N}$ atoms. Both the $\mathrm{B} \cdots \mathrm{N}$ and $\mathrm{H}-\mathrm{H}$ distances for this $\mathrm{H}_{2}$ were elongated compared to their normal values, toward distances calculated for the transition state in solution heterolysis. ${ }^{31}$

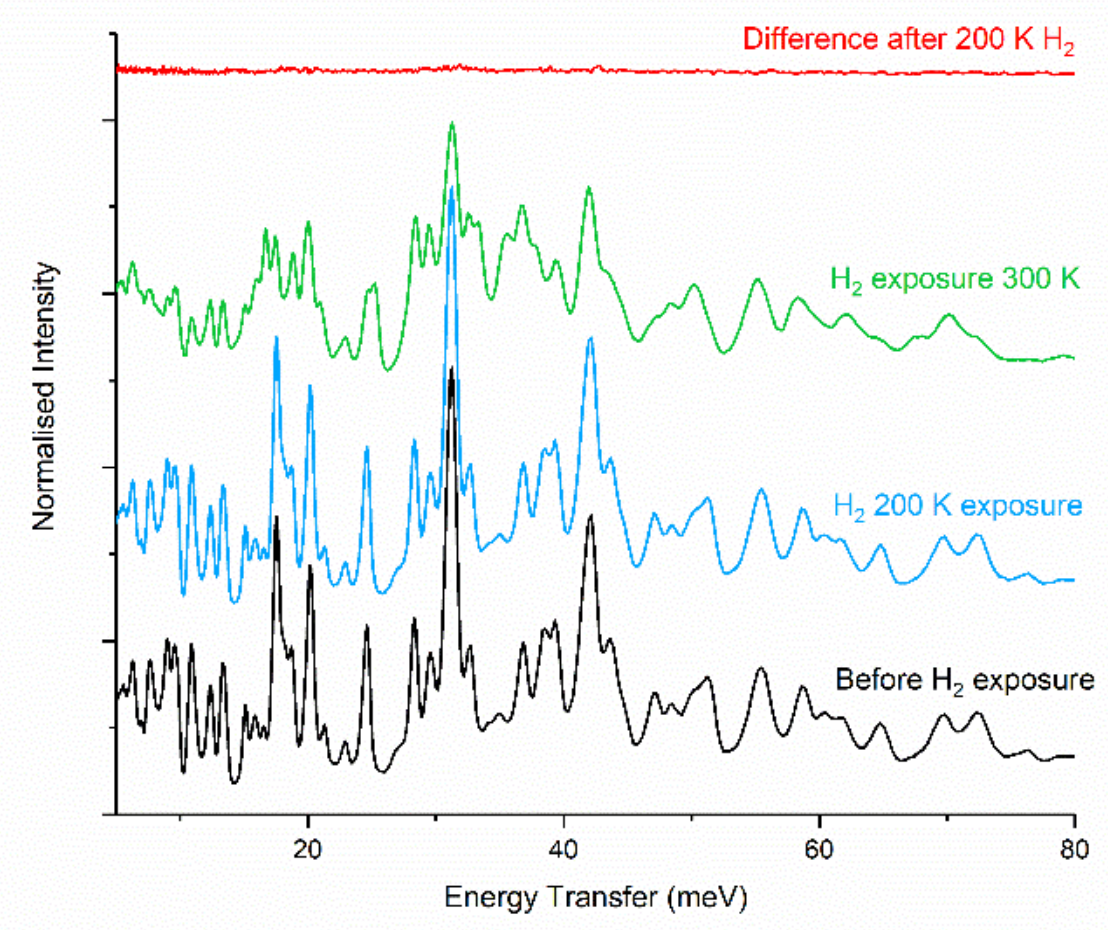

Figure 4. INS spectra at $10 \mathrm{~K}$ before (black) and after exposure to $\mathrm{H}_{2}$ gas at 200 (blue) and $300 \mathrm{~K}$ (green). The difference after exposure to $\mathrm{H}_{2}$ at $200 \mathrm{~K}$ is shown at top.

INS spectra were calculated both from the harmonic modes of the quantum mechanical optimized structure with and without $\mathrm{H}_{2}$, as well as Ab Initio Molecular Dynamics (AIMD) simulations that allow for anharmonic treatment of the frequencies. Both approaches indicated that the presence of $\mathrm{H}_{2}$ within the lattice would lead to significant changes in the INS spectra (Figs. S8 and S13). Our experimental results (Fig. 4), which did not detect changes, therefore lead us to believe that the concentration of $\mathrm{H}_{2}$ in the lattice is much lower than the stoichiometric quantity used in the calculations. The MD simulations also indicated 
that $\mathrm{H}_{2}$ has significant mobility within the KCAT lattice. Each of the four sites traversed a large volume within the lattice during the 12 ps simulation, up to $5 \AA$ from the original starting location (Figs. S11 and S12). These diffuse volumes are consistent with shallow minima that $\mathrm{H}_{2}$ can access near $300 \mathrm{~K}$.

Table 1. Calculated electronic energy changes and distances to nearest $\mathrm{B}$ and $\mathrm{N}$ atoms for addition of molecular $\mathrm{H}_{2}$ in different sites within crystalline KCAT.

\begin{tabular}{cccccc}
\hline $\mathrm{H}_{2}$ site & $\begin{array}{c}\triangle \mathrm{E} \\
(\mathrm{kJ} \mathrm{mol}-1)\end{array}$ & $\begin{array}{c}\mathrm{B} \cdots \mathrm{H} \text { distance } \\
(\AA)\end{array}$ & $\begin{array}{c}\mathrm{N} \cdots \mathrm{H} \text { distance } \\
(\AA)\end{array}$ & $\begin{array}{c}\mathrm{B} \cdots \mathrm{N} \text { distance } \\
(\AA)\end{array}$ & $\begin{array}{c}\mathrm{H}-\mathrm{H} \text { distance } \\
(\AA)\end{array}$ \\
\hline 1 & 26 & 5.10 & 4.72 & 3.07 & 0.75 \\
2 & 32 & 2.39 & 2.15 & 3.14 & 0.76 \\
3 & 39 & 2.52 & 2.35 & 3.05 & 0.75 \\
4 & 36 & 6.67 & 5.25 & 3.05 & 0.75 \\
\hline
\end{tabular}

\section{Temperature Programmed Desorption}

The presence of molecular $\mathrm{H}_{2}$ in the crystal was also sought using temperature programmed desorption (TPD). $1 \mathrm{mg}$ of KCAT was exposed to $1 \mathrm{~atm}$ of $\mathrm{H}_{2}$ gas for 30 minutes at $298 \mathrm{~K}$ and cooled rapidly to $77 \mathrm{~K}$ under the $\mathrm{H}_{2}$ atmosphere. Our previous microscopy and INS experiments indicated that $\mathrm{H}_{2}$ enters the crystal at $298 \mathrm{~K}$ but not at $77 \mathrm{~K}$. The sample was pumped to ca. $1 \times 10^{-8}$ Torr at $77 \mathrm{~K}$ before heating at $5 \mathrm{~K} / \mathrm{min}$ to $330 \mathrm{~K}$ and monitoring any gas liberated with a quadrupole mass spectrometer. Despite the sensitivity of this experiment to small ( $\geq 0.01$ wt. \%) quantities of gas, no $\mathrm{H}_{2}$ was detected during the temperature increase (Fig. S7) and the crystals retained their bright color at the end of the experiment.

\section{Discussion}

Activation or heterolysis of molecular $\mathrm{H}_{2}$ to a proton and a hydride, $\mathrm{H}^{+} / \mathrm{H}^{-}$, by solid phase KCAT at ambient temperature and pressure is readily detected by the visual bleaching of the brightly colored FLP. The heterolysis was confirmed by several analytical methods and takes place as a single crystal to single crystal transformation. This is apparent from visual observation; for example, Fig. 3 shows a small number of crystals whose external appearance is unchanged as the heterolysis takes place within their interior. Further, a single crystal of KCATH2 suitable for structure determination was readily obtained from KCAT crystals exposed to $\mathrm{H}_{2}$ gas. The color change suggests that formation of 
KCATH2 takes place in the solid state rather than during dissolution in the NMR solvent, for example. This was confirmed by the INS experiments, where the ion pair was observed after reaction only of the solid KCAT and gaseous hydrogen (Fig. 4). The heterolysis is more exergonic in the solid state $\left(\Delta A_{\text {calc }}=-125 \mathrm{~kJ} \mathrm{~mol}^{-1}\right)$ than in solution $\left(\Delta G_{\text {calc }}=-50 \mathrm{~kJ} \mathrm{~mol}^{-1}\right) .{ }^{31}$ The reason for this was not examined but is likely due to electrostatic stabilization between the zwitterionic charges in the closely packed crystalline KCATH2. Benchmarking computational studies by Grimme et al.32 have also reported additional stabilization in solid-state FLPs for $\mathrm{H}_{2}$ hydrolysis, however their observation was that the stabilization in intra-molecular FLPs is up to $\sim 20 \mathrm{~kJ} \mathrm{~mol}^{-1}$. We calculate a significantly larger difference of $\sim 75 \mathrm{~kJ} \mathrm{~mol}^{-1}$., closer to the value Grimme reported for inter-molecular FLPs. We attribute this additional stabilization to the different packing of KCAT and KCATH2, in contrast to the earlier study ${ }^{32}$ where only the structure of the FLP is used. This difference suggests that in solid state FLPs, in addition to the crystal field effects, packing can have significant impact on the thermochemistry of the reaction. We considered two potential mechanisms for the phase transition of KCAT to KCATH2: (i) a diffusion mechanism where gaseous $\mathrm{H}_{2}$ diffuses into crystalline KCAT followed by the activated heterolysis of $\mathrm{H}_{2}$ and (ii) a decrepitation mechanism where the outside of the solid phase particle reacts with gaseous $\mathrm{H}_{2}$ and peels away layer by layer until complete conversion of the FLP to the corresponding ion pair has occurred. Optical microscopy of the FLP crystallites eliminated the possibility of a decrepitation mechanism, and this observation was supported by the small lattice expansion of 2.1 vol.\% on hydrogen uptake observed by diffraction. This may be compared to classical decrepitation systems, such as metal hydrides and Nd-Fe-B, which show expansions in the range of 10-15 vol.\%.33, 34 The lattice expansion is, however, consistent with those reported for organometallic SC-SC transformations. ${ }^{35}$ As a consequence of these observations, we assign a diffusion mechanism for the phase transformation in crystalline KCAT. The rate of diffusion is faster than, or at least comparable to, the rate of $\mathrm{H}_{2}$ heterolysis. If the rate of heterolysis was significantly faster than diffusion, we would expect to see a more clearly defined skin of white KCATH2 on the exterior of the crystals, much like the CO complex observed by Pike et al. ${ }^{19}$ on the surface of their Rh butadiene complex. However, our optical observations revealed a more widespread and diffuse color change without a sharply defined boundary.

Little has been reported on the absolute rates of $\mathrm{H}_{2}$ activation by FLPs in solution, and there are no reports on the kinetics of solid phase FLPs reacting with gaseous $\mathrm{H}_{2}$. We assume the reaction to involve an activated process that requires near ambient temperature. This conclusion is based on observations at temperatures $<200 \mathrm{~K}$ that the ion pair was not formed, even under a high pressure of $\mathrm{H}_{2}$ gas in our in-situ INS measurements.

We hypothesized that a diffusion mechanism offers an opportunity to observe the precursor complex of KCAT with molecular hydrogen, $\left\{\mathrm{H}_{2}---\mathrm{KCAT}(\mathrm{s})\right\}$, where the $\mathrm{H}_{2}$ is energetically stabilized within the cavity of the FLP to form a polarized $\mathrm{H}_{2}$ molecule: 
$\mathrm{H}_{2}(\mathrm{~g})+\mathrm{KCAT}(\mathrm{s}) \Leftrightarrow\left\{\mathrm{H}_{2}--\mathrm{KCAT}(\mathrm{s})\right\} \Leftrightarrow \operatorname{KCATH} 2(\mathrm{~s})$

We sought spectroscopic evidence for such a precursor complex with INS spectroscopy, encouraged by calculations (Figs. S8, S13) that indicated discernable differences in the spectra would arise from the presence of $\mathrm{H}_{2}$ within the lattice. We considered that KCAT possessed several features that might be advantageous for the formation of such a complex: (i) a linked FLP with the Lewis acid and base on the same framework, (ii) a geometry with the Lewis acidic B and Lewis basic N fixed about $3 \AA$ Apart, (iii) a strong Lewis acid and base pair that would provide a large electric field to polarize $\mathrm{H}_{2}{ }^{29}, 36$ and (iv) a favorable equilibrium toward the zwitterion suggesting a relatively low activation energy for a precursor complex. However, despite these favorable factors, no evidence for an intermediate phase was found by INS.

The INS results were supported by calculations of the energetic penalty for molecular $\mathrm{H}_{2}$ to reside in the KCAT crystal. The relative stabilities of $\mathrm{H}_{2}$ occupying different positions in the crystal showed that the most stable site was not productive for $\mathrm{H}_{2}$ activation, since $\mathrm{H}_{2}$ was more than $6 \AA$ away from the nearest $\mathrm{B} \cdots \mathrm{N}$ site. This is consistent with the $\mathrm{H}_{2}$ accessing the active site at higher temperatures, but not at low temperatures. This observation is reminiscent of work by Weller et al., ${ }^{37}$ on Rh based MOFs where Xe gas was found to diffuse away from the active site and reside in hydrophobic pockets, similar to enzymatic gas channels. Further, although the formation of the ion pair clearly showed that $\mathrm{H}_{2}$ had entered the solid at room temperature, our TPD measurements did not detect $\mathrm{H}_{2}$ diffusing out of the lattice after freezing the sample to $77 \mathrm{~K}$ and evacuating the surrounding gas. INS demonstrated heterolysis does not occur at this low temperature, and so we anticipated that $\mathrm{H}_{2}$ would be trapped inside the crystal. Upon heating, the low pressure of $\mathrm{H}_{2}$ outside the crystal would reverse diffusion as the temperature was raised.

All these results indicated that the concentration of $\mathrm{H}_{2}$ within the KCAT crystal was small. The Kitaigorodsky packing index, calculated with PLATON, provides a measure of the free space within a crystal based on van der Waals radii. At $300 \mathrm{~K}$, KCAT has a packing index of $67 \%$, which is within the range of $65-77 \%$ found in most crystals. ${ }^{38}$ Although this is near the lower bound, it indicates there is no reason to expect an unusually high concentration of $\mathrm{H}_{2}$ in KCAT compared to other compounds.

The reaction path for heterolysis by molecular KCAT in solution has been calculated by Repo et al. ${ }^{31}$ This work did not identify a precursor complex, but a transition state with an activation barrier of $74 \mathrm{~kJ} \mathrm{~mol}^{-1}$ where the $\mathrm{B} \cdots \mathrm{N}$ distance is elongated to $3.23 \AA$ and the $\mathrm{H}-\mathrm{H}$ distance to $0.76 \AA$ A. Our calculations (site 2, Table 1) also showed a state with an elongated 
$\mathrm{H}-\mathrm{H}$ bond and with a more modest elongation of the $\mathrm{B} \cdots \mathrm{N}$ distance to $3.14 \AA$, likely restricted by the packing of the crystal. Although not formally identified as a transition state, it is interesting to note that the geometry of the $\mathrm{H}_{2}$ and nearby KCAT molecules in our calculations are nearly identical to those in solution and that the energy for $\mathrm{H}_{2}$ addition in the solid state is smaller than the activation barrier in solution, at $50 \mathrm{~kJ} \mathrm{~mol}^{-1}$ compared to the reactants.

The observation of multiple calculated minima where the $\mathrm{H}_{2}$ can reside in the FLP lattice indicates that $\mathrm{H}_{2}$ may get trapped in unfavorable positions at low temperatures, inhibiting access to the reactive pocket. Equally, such conclusions suggest that although $\mathrm{H}_{2}$ diffuses relatively rapidly into the lattice, the rate limiting step for heterolysis of molecular $\mathrm{H}_{2}$ within KCAT is local movement to the reactive site rather than the molecular rearrangement of the FLP in the solid state. In this light, an interesting follow up could focus on studying the kinetics of the reaction using solid-state (e.g. Avrami) models, including the use of $\mathrm{D}_{2}$ to distinguish diffusion from activated rate-limiting processes.

\section{Conclusions}

We have demonstrated the first heterolysis of hydrogen in an intramolecular, metal-free FLP system in the solid state. Hydrogen diffuses into the crystal lattice of KCAT at $298 \mathrm{~K}$ but remains at concentrations too low for detection by inelastic neutron scattering or temperature programmed desorption. Heterolysis occurs at $298 \mathrm{~K}$ to form the zwitterion in a single crystal - single crystal transformation with a strong exergonic free energy change. Observation of the color change associated with this reaction shows that diffusion within the KCAT crystal occurs more rapidly than heterolysis at a local site takes place. This is likely to be a consequence of $\mathrm{H}_{2}$ moving between and occupying sites not closely associated with the frustrated $\mathrm{B} \cdots \mathrm{N}$ to a greater extent than the sites proximate to $\mathrm{B} \cdots \mathrm{N}$.

\section{Acknowledgements}

The authors (MEB, BG, AJK, GKS and TA) gratefully acknowledge support from U.S. Department of Energy (DOE), Office of Science, Office of Basic Energy Sciences, Division of Chemical Sciences, Geosciences \& Biosciences for providing the impetus for this research. SM was supported by DOE, Office of Science, Office of Workforce Development for Teachers and Scientists under the Science Undergraduate Laboratory Internships program. A portion of the research including some computation was performed using EMSL (grid.436923.9), a DOE Office of Science User Facility sponsored by the Office of Biological and Environmental Research. Further computing resources were provided by PNNL 
Research Computing. The VISION experiments used resources at the Spallation Neutron Source, a DOE Office of Science User Facility operated by the Oak Ridge National Laboratory. TOSCA experiments at the ISIS Neutron and Muon Source were supported by a beamtime allocation RB1510079 from the Science and Technology Facilities Council. KC and TR thank the Academy of Finland (project \# 276586) for financial support.

\section{ASSOCIATED CONTENT}

Supporting Information Available: Experimental and computational details. Powder XRD results, crystallographic tables and discussion of solvated and solvent-free KCATH2. VISION INS spectra at temperatures between 10 and $200 \mathrm{~K}$, TOSCA INS spectra, TPD traces. Calculated INS spectra and images of optimized and dynamic $\mathrm{H}_{2}$ positions within KCAT lattice.

Crystallographic data for KCATH2 have been deposited with the Cambridge Crystallographic Data Centre with deposition number 2020667.

\section{References}

1. Chen, Q. A.; Ye, Z. S.; Duan, Y.; Zhou, Y. G., Homogeneous palladium-catalyzed asymmetric hydrogenation. Chem Soc Rev 2013, 42 (2), 497-511.

2. Rakowski DuBois, M.; DuBois, D. L., The roles of the first and second coordination spheres in the design of molecular catalysts for $\mathrm{H} 2$ production and oxidation. Chem Soc Rev 2009, 38 (1), 62-72.

3. Alig, L.; Fritz, M.; Schneider, S., First-Row Transition Metal (De)Hydrogenation Catalysis Based On Functional Pincer Ligands. Chem Rev 2019, 119 (4), 2681-2751.

4. Filonenko, G. A.; van Putten, R.; Hensen, E. J. M.; Pidko, E. A., Catalytic (de)hydrogenation promoted by non-precious metals - Co, Fe and $\mathrm{Mn}$ : recent advances in an emerging field. Chem Soc Rev 2018, 47 (4), 1459-1483.

5. Belkova, N. V.; Epstein, L. M.; Filippov, O. A.; Shubina, E. S., Hydrogen and Dihydrogen Bonds in the Reactions of Metal Hydrides. Chem Rev 2016, 116 (15), 8545-87.

6. Stephan, D. W., Catalysis, FLPs, and Beyond. Chem 2020.

7. Xing, J. Y.; Buffet, J. C.; Rees, N. H.; Norby, P.; O'Hare, D., Hydrogen cleavage by solid-phase frustrated Lewis pairs. Chem Commun 2016, 52 (69), 10478-10481. 
8. Ma, Y.; Zhang, S.; Chang, C. R.; Huang, Z. Q.; Ho, J. C.; Qu, Y., Semi-solid and solid frustrated Lewis pair catalysts. Chem Soc Rev 2018, 47 (15), 5541-5553.

9. Ye, J. Y.; Johnson, J. K., Design of Lewis Pair-Functionalized Metal Organic Frameworks for CO2 Hydrogenation. Acs Catal 2015, 5 (5), 2921-2928.

10. Ye, J. Y.; Johnson, J. K., Catalytic hydrogenation of $\mathrm{CO} 2$ to methanol in a Lewis pair functionalized MOF. Catal Sci Technol 2016, 6 (24), 8392-8405.

11. Heshmat, M., Alternative Pathway of $\mathrm{CO} 2$ Hydrogenation by Lewis-Pair-Functionalized UiO-66 MOF Revealed by Metadynamics Simulations. J Phys Chem C 2020, 124 (20), 10951-10960.

12. Wang, L.; Kehr, G.; Daniliuc, C. G.; Brinkkotter, M.; Wiegand, T.; Wubker, A. L.; Eckert, H.; Liu, L.; Brandenburg, J. G.; Grimme, S.; Erker, G., Solid state frustrated Lewis pair chemistry. Chem Sci 2018, 9 (21), 4859-4865.

13. Ghuman, K. K.; Hoch, L. B.; Wood, T. E.; Mims, C.; Singh, C. V.; Ozin, G. A., Surface Analogues of Molecular Frustrated Lewis Pairs in : Heterogeneous CO2 Hydrogenation Catalysis. Acs Catal 2016, 6 (9), 5764-5770.

14. Wang, L.; Yan, T.; Song, R.; Sun, W.; Dong, Y.; Guo, J.; Zhang, Z.; Wang, X.; Ozin, G. A., RoomTemperature Activation of $\mathrm{H} 2$ by a Surface Frustrated Lewis Pair. Angewandte Chemie International Edition 2019, 58 (28), 9501-9505.

15. Houghton, A. Y.; Autrey, T., Calorimetric Study of the Activation of Hydrogen by Tris(pentafluorophenyl)borane and Trimesitylphosphine. The Journal of Physical Chemistry A 2017, 121 (46), 8785-8790.

16. Whittemore, S. M.; Autrey, T., Kinetic and Thermodynamic Study of the Reduction of 1,1Diphenylethylene by a Thermally Frustrated Diethyl Ether-BCF Lewis Pair. Israel Journal of Chemistry 2015, 55 (2), 196-201.

17. Huang, Z.; White, P. S.; Brookhart, M., Ligand exchanges and selective catalytic hydrogenation in molecular single crystals. Nature 2010, 465 (7298), 598-601.

18. Bianchini, C.; Farnetti, E.; Graziani, M.; Kaspar, J.; Vizza, F., Molecular Solid-State Organometallic Chemistry of Tripodal (Polyphosphine)Metal Complexes - Catalytic-Hydrogenation of Ethylene at Iridium. J Am Chem Soc 1993, 115 (5), 1753-1759.

19. Pike, S. D.; Kramer, T.; Rees, N. H.; Macgregor, S. A.; Weller, A. S., Stoichiometric and Catalytic Solid-Gas Reactivity of Rhodium Bisphosphine Complexes. Organometallics 2015, 34 (8), 1487-1497.

20. Pike, S. D.; Chadwick, F. M.; Rees, N. H.; Scott, M. P.; Weller, A. S.; Kramer, T.; Macgregor, S. A., Solid-state synthesis and characterization of sigma-alkane complexes, [Rh(L2)(eta(2),eta(2)C7H12)][BAr(F)4] (L2 = bidentate chelating phosphine). J Am Chem Soc 2015, 137 (2), 820-33.

21. Chadwick, F. M.; Kramer, T.; Gutmann, T.; Rees, N. H.; Thompson, A. L.; Edwards, A. J.; Buntkowsky, G.; Macgregor, S. A.; Weller, A. S., Selective C-H Activation at a Molecular Rhodium SigmaAlkane Complex by Solid/Gas Single-Crystal to Single-Crystal H/D Exchange. J Am Chem Soc 2016, 138 (40), 13369-13378.

22. Chernichenko, K.; Nieger, M.; Leskela, M.; Repo, T., Hydrogen activation by 2-boryl-N,Ndialkylanilines: a revision of Piers' ansa-aminoborane. Dalton T 2012, 41 (30), 9029-9032.

23. Camaioni, D. M.; Ginovska-Pangovska, B.; Schenter, G. K.; Kathmann, S. M.; Autrey, T., Analysis of the Activation and Heterolytic Dissociation of $\mathrm{H}-2$ by Frustrated Lewis Pairs: NH3/BX3 $(\mathrm{X}=\mathrm{H}$, $\mathrm{F}$, and Cl). Journal of Physical Chemistry A 2012, 116 (26), 7228-7237.

24. $\mathrm{Pu}, \mathrm{M}$. P.; Privalov, T., Ab initio dynamics trajectory study of the heterolytic cleavage of $\mathrm{H}-2$ by a Lewis acid $[\mathrm{B}(\mathrm{C6F5})(3)]$ and a Lewis base $[\mathrm{P}(\mathrm{tBu})(3)]$. J Chem Phys 2013, 138 (15).

25. Wu, D.; Jia, D.; Liu, L.; Zhang, L.; Guo, J., Reactivity of 2,6-lutidine/BR(3) and pyridine/BR(3) Lewis pairs $(R=F, M e, C(6) F(5))$ : a density functional study. J Phys Chem A 2010, 114 (43), 11738-45. 
26. VandeVondele, J.; Krack, M.; Mohamed, F.; Parrinello, M.; Chassaing, T.; Hutter, J., QUICKSTEP: Fast and accurate density functional calculations using a mixed Gaussian and plane waves approach. Comput Phys Commun 2005, 167 (2), 103-128.

27. Perdew, J. P.; Burke, K.; Ernzerhof, M., Generalized gradient approximation made simple. Phys Rev Lett 1996, 77 (18), 3865-3868.

28. Grimme, S., Semiempirical GGA-type density functional constructed with a long-range dispersion correction. J Comput Chem 2006, 27 (15), 1787-1799.

29. Grimme, S.; Kruse, H.; Goerigk, L.; Erker, G., The Mechanism of Dihydrogen Activation by Frustrated Lewis Pairs Revisited. Angew Chem Int Edit 2010, 49 (8), 1402-1405.

30. Goedecker, S.; Teter, M.; Hutter, J., Separable dual-space Gaussian pseudopotentials. Phys Rev B 1996, 54 (3), 1703-1710.

31. Chernichenko, K.; Kotai, B.; Nieger, M.; Heikkinen, S.; Papai, I.; Repo, T., Replacing C6F5 groups with $\mathrm{Cl}$ and $\mathrm{H}$ atoms in frustrated Lewis pairs: $\mathrm{H}-2$ additions and catalytic hydrogenations. Dalton T 2017, 46 (7), 2263-2269.

32. Liu, L.; Brandenburg, J. G.; Grimme, S., On the hydrogen activation by frustrated Lewis pairs in the solid state: benchmark studies and theoretical insights. Philos TR Soc A 2017, 375 (2101).

33. Joubert, J. M.; Latroche, M.; Cerny, R.; Percheron-Guegan, A.; Yvon, K., Hydrogen cycling induced degradation in LaNi5-type materials. J Alloy Compd 2002, 330, 208-214.

34. Liszkowski, P.; Turek, K.; Figiel, H., The influence of decrepitation on the diffusion kinetics of hydrogen in Nd-Fe-B. J Alloy Compd 2000, 307, 297-303.

35. Pike, S. D.; Weller, A. S., Organometallic synthesis, reactivity and catalysis in the solid state using well-defined single-site species. Philos Trans A Math Phys Eng Sci 2015, 373 (2037).

36. Rokob, T. A.; Bako, I.; Stirling, A.; Hamza, A.; Papai, I., Reactivity Models of Hydrogen Activation by Frustrated Lewis Pairs: Synergistic Electron Transfers or Polarization by Electric Field? J Am Chem Soc 2013, 135 (11), 4425-4437.

37. Martinez-Martinez, A. J.; Rees, N. H.; Weller, A. S., Reversible Encapsulation of Xenon and CH2 $\mathrm{Cl} 2$ in a Solid-State Molecular Organometallic Framework (Guest@SMOM). Angew Chem Int Ed Engl 2019, 58 (47), 16873-16877.

38. Kitaigorodsky, A. I., Molecular Crystals and Molecules. Academic Press: New York, 1973. 


\section{TOC Graphic}

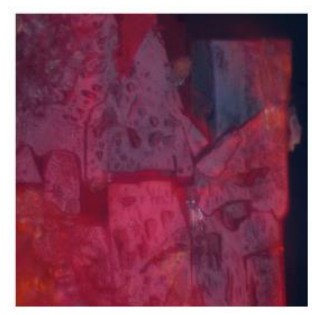

Single crystal $\rightarrow$ Single crystal<smiles>CC1(C)CCCC(C)(C)N1c1ccccc1P(C)(=O)C1CCCCC1</smiles>

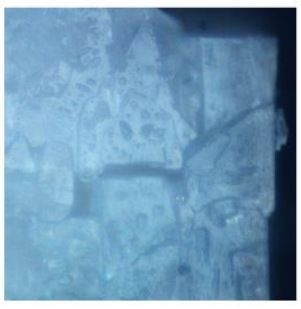

$\mathrm{H}_{2}$ gas

1 atmosphere

Room temperature

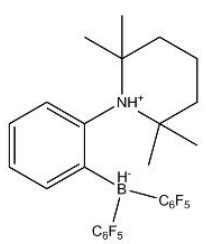

Synopsis: A crystalline intramolecular frustrated Lewis acid-base pair (FLP) reacts with gaseous hydrogen under ambient conditions, breaking the $\mathrm{H}-\mathrm{H}$ bond and forming a $\mathrm{H}^{+} / \mathrm{H}^{-}$ zwitterionic pair. This single crystal to single crystal transformation provides the first demonstration of a non-metal example of $\mathrm{H}_{2}$ heterolysis by a unimolecular FLP in the solid state, of relevance to catalysis of energy carriers. 